\title{
High Reliability Organizing (HRO) is the Extension of Neonatology during Pandemic COVID-19
}

Daved van Stralen, MD, FAAP, Thomas A. Mercer, RAdm, USN

\section{Abstract}

Neonatology began with the extension of care to smaller babies born earlier in gestation and now extending into the threat of $\mathrm{CO}-$ VID-1. Extending operations into a new environment places everyone in novel situations. Emerging from actual operations within dangerous contexts, HRO extends operations and the organization into uncertain, adverse, and hostile environments. The physi$\mathrm{cal}$ actions of care come from motor cognition, the influence of the cerebellum and motor cortex on cognition, and how we learn through physical action to understand events. Rather than study the myriad ways a system can fail or how it can reach the final common pathway of failing, we can study and respond to it as a liminal zone. It is common to all failures - an exigency of ambiguous information, impending proximal threat, uncertainty, and demand for action. Engagement in the liminal zone is the same as engagement for extension, drawing from four domains: categorization, methods of decision making, the significance of the affective processes, and modulation of stress and fear responses. Perhaps the purpose of safety, reliability, and resilience is not to safely work in a dangerous context but to support the extension of the organization into uncertainty, a new environment, unpredictable circumstances, or to penetrate threats. When a person believes in themselves, they will leave their place of safety and engage the unknown when they know they are supported. This is the extension of neonatology.

\section{"The continuing extension of neonatology now includes surgical care, complex mechanical ventilation, and pulmonary care, support for families, and more. "}

\section{Introduction}

What falls under neonatology? The discipline of neonatology started as the extension of care to smaller babies born earlier in gestation. That, alone, is commendable. But the characteristics of the Neonatologist and neonatal nurses extended neonatology to babies with respiratory disease, and respiratory care practitioners brought their expertise. The continuing extension of neonatology now includes surgical care, complex mechanical ventilation, and pulmonary care, support for families, and more.

The extension of neonatology asserted effective care over more difficult physiology, dealing with low oxygen values and low temperatures, somewhat aligned with the problems of early high altitude climbers in the Himalaya (1). Climbers gained altitude, but it was the synthesis of science and climbing, created by an indi- vidual with experience in both domains, that brought them to the top of Mount Everest. Similarly, Neonatologists with experience in both domains of science and bedside medical care have gained life for those newborns who would have died and for those who would have suffered a severe disability.

The extension of neonatology into the threat of COVID-19 is the most recent expansion of neonatology. Neonatology demonstrates many of the characteristics of High-Reliability Organizing (HRO). The problem common to organizations seeking greater effectiveness is the translation of HRO characteristics to neonatology. Mistranslations and misunderstandings undermine the benefits of $\mathrm{HRO}$ as a method of extension into new realms (2).

\section{"The problem common to organizations seeking greater effectiveness is the translation of HRO characteristics to neonatology. Mistranslations and misunderstandings undermine the benefits of HRO as a method of extension into new} realms (2)."

Dangerous contexts remain dangerous. We commend the outside observer who suggests bringing order and creating structure through design and command. The outside vantage point gives a better view of identifying discrete principles, reducing mistakes, and stopping errors. Organizing the confused, dangerous work environment from the outside seems prudent. Outside observers, however, miss the meaning of weak yet salient signals and the power of subtle or nuanced actions taken at the right time.

An observer, removed from the problem, evaluates the situation with theory and scientific rationality. The operator, engaged in the turbulence of events, becomes part of the problem (2). The operator learns what works through action; experienced operators think best while acting $(3,4)$. Karl Weick captured this in his "recipe" for sensemaking, "How can I know what I think until I see what I say?" (5). Other distinctions in thinking between observers and operators include the use of abstractions over concreteness and nouns versus verbs, respectively $(2,6-12)$. We see this in Weick's distinction between High-Reliability Organization versus High-Reliability Organizing (10).

Advances in neuroscience provide support for several characteristics found in dangerous contexts -- for example, language processing and the link between cognition and action. This is not a trite digression. There is order within a difficult period, but it is likely more pragmatic than normative, and the nature of that order

NEONATOLOGY TODAY is interested in publishing manuscripts from Neonatologists, Fellows, NNPs and those involved in caring for neonates on case studies, research results, hospital news, meeting announcements, and other pertinent topics.

Please submit your manuscript to: LomaLindaPublishingCompany@gmail.com 
is rendered less accurately by the concepts of a spectator than by the detailed acquaintance of the insider (2). Unrecognized by operators and invisible to spectators is the influence of action on cognition. The extension of neonatology to gain life emerged from Neonatologists physically engaged in care at the bedside. The Captain of a US Navy nuclear aircraft carrier is on the bridge for all dynamic operations (TAM). It is the intimate experience of problem-solving on the fly where we experience what Weick (personal communication) describes as "the clash between a mistaken old belief and an updated belief." That is the extension of an organization into uncertainty rather than laboratory studies and evidencebased knowledge.

Extending operations into a new environment places everyone in novel situations. Motor cognition describes how we adjust our actions to changing situations and learn through physical actions. New encounters increase the possibility of failing but, past a threshold, the environment of failing is shared amongst all causations and situations. Developing the skill of responding to the final common pathway of failing supports extension and learning. Nothing is entirely new; knowledge and experience from an environment with some shared similarities initiates extension from our capabilities. Extension through the final common pathway draws upon four domains of engagement that differ from how these domains are used in stable environments (categorization, methods of decision making, the significance of the affective processes, and modulation of the stress and fear responses). We end with some observations on hidden impairments to extension. They seem to help but will block the extension of individuals, teams, groups, and organizations.

\section{"Extension through the final common pathway draws upon four domains of engagement that differ from how these domains are used in stable environments (categorization, methods of decision making, the significance of the affective processes, and modulation of the stress and fear responses)."}

\section{The Motor Cognition of Extension}

"Whenever movement follows unhesitatingly and immediately the notion of it in mind, we have ideo-motor action. We are then aware of nothing between the conception and the execution," William James (13).

The historical roots of motor cognition are the pragmatist school of philosophy and William James (14). Motor cognition is action representation for voluntary action. Their perceivable effects represent actions under our control. In fact, the intent or thinking of the effects in mind is sufficient to act immediately (15). Cognitive processes for action include the urge and intention to act and adjust those actions while acting immediately. A mirror system allows us to understand the intent and actions of others, creating a gateway to social cognition. Mirror systems have motor output, differentiating them from visual perception processing (14).

HRO operations have common elements with motor cognition. During an emergency, we focus on the consequences of our actions and bottom-up feedback (2). This is similar to pragmatist philosophy and represented in motor cognition by perceivable ef- fects as bottom-up feedback. $(14,15)$. Mirror systems support the heedful interrelating and trust found on an aircraft carrier flight deck (16), physical rescue in the field (8), or neonatal resuscitation.

The Mirror system is how we learn by watching others (17) and figure out their intent without speaking (18). Because the mirror system neurons are in the pre-motor cortex, they do not respond unless action is executable. Through the mirror system, we can communicate to others by our actions, a process called communicative intention (19).

The academic background of the Neonatologist supports the routine use of abstract thought while operations with uncertainty or adversity in a liminal environment demand accurate, concrete descriptions $(1,3)$. This has neuroanatomic consequences. The sensorimotor neural network processes sentences with concrete nouns and words and abstract words but with a preference for concrete terms. The linguistic system preferentially processes abstract nouns and verbs (20). For motor versus visual abstract words, abstract motor words will specifically activate motor areas while visual abstract words specifically elicit higher visual area activity (21). The use of concrete, active words facilitates action, while abstract words tend to generate thinking. This seems prudent until you realize the brain's response to stress constrains executive functions and impairs abstract thought (22). During an emergency, abstract words send messages to areas of the brain impaired by stress.

Operators in dangerous contexts use concrete nouns for description and emphasize action verbs for communication. Recent neuroscience findings support this behavior. Action words and motor action noted above share common cortical representations. Action verbs, more so than concrete nouns, affect overt motor performance dependent on timing. That is, an action verb will interfere with a reaching movement in progress within $200 \mathrm{msec}$. The same words processed before movement will assist the movement (23). This action, fortunately, is category-specific. A quick shout to move a hand causes hands to move and not random body parts. The category-specific, functional linking of language and motor action in the left hemispheric cortical systems link arm and leg actions with the processing of specific kinds of words. The two systems interact to produce meaningful information about language and action (24-26). "We assume the process to be a continuing one - more organizing than an organization," Karl Weick (10). The distinction of organizing (verb) vs. organization (noun) is one of the twelve most frequently cited concepts on organizing from Weick (27). The brain processes verbs faster than nouns.

The cerebellum and motor cortex also influence cognition. Executive functions and higher-level cognitive cortical functions draw upon interactions with cerebellar motor functions (28-30). Highlevel knowledge is grounded in sensory and motor experience (28). This shapes the motor system on anticipation and provides information for the meaning of potential action $(26,31)$. We rely on reciprocal feedback from the environment (8). We think by acting (4).

Because uncertainty, adverse situations, and hostile environments have different environmental geometry, they require a different engagement geometry. Circumstances may change during sensemaking or acting, and your mind must keep up. The time course described is not necessarily minutes but could be days. Time compression matches the time horizon, minutes for an active resuscitation, weeks for a growing, healing lung. The problem is how we mentally map events and our responses. We are taught by organizing knowledge and concepts in hierarchies that show relationships and connect terms with measurable distances between points. This is a Euclidean space. There is growth but no movement. 
The circumstances during COVID-19 and neonatology as a discipline are in a continuously changing actual world. Our team's actions are continuous, independent, and interdependent. This geometry describes a scale-free topology (32). The central concept of topology is continuity, a notion of nearness that is preserved by a continuous function. Topology does not localize an object in Euclidean space, allowing a discipline to extend beyond Euclidean restrictions (33). The geometry is preserved under continuous motions because distance and dimension are not relevant. In neonatology, operations occur in different kinds of space, the distance between objects is a matter of function of the relations, and differences between objects are a matter of variety in the relations (33). Topology provides a language of continuity that is general enough to be developed in new ways. Topology extends neonatology beyond the restrictions of Euclidean space. The major extensions of neonatology have occurred through topological space rather than Euclidean space.

\section{"Topology extends neonatology beyond the restrictions of Euclidean space. The major extensions of neonatology have occurred through topological space rather than Euclidean space."}

Karl Weick's 'sensemaking recipe' now applies to these topological events: "How can I know what I think until I see what I say?" (5). We can distinguish the neuroscience of his recipe.

I can't know what I think until I act. Intention cannot cause our actions because conscious intention occurs after preparatory brain activity in the frontal and parietal brain areas (34). It would make sense that purposive action derives from intention, which would mediate between cognitive desires and purposive motor behavior. Rather than mediating, the two distinct brain operations, cognitive intention and motor behavior, must coordinate. This is the "Interface Problem," made difficult because of the importance of motor representations in creating purposive behavior (35).

The subjective experience of conscious intention often contains two components: a sense of urgency, or being about to move, and a reference forward to the goal object or event [22]. Our perceptions help us recognize whether a response was due to our own actions, giving us a sense of agency (34). Without action, we do not gain a sense of agency.

Actions create what we think, which continuously changes until we finish acting. During our behavioral interactions with the environment, our brain specifies desirable actions as the environment changes (36). Continuous, bottom-up feedback for sensorimotor control, through the motor system, detects prediction error, updating ongoing action. This feedback enhances or cancels some sensorimotor signals. Self-generated cancellation as a motor function also explains why we cannot tickle ourselves. It is due to the sensory feedback through the motor system (37). Alternative actions continue to be mentally processed (36). This may extend to language comprehension, social cognition (38), and interpreting sensory signals (39). This makes visible to ourselves our intention.

"The clash between a mistaken old belief and an updated belief would seem to be a form of dissonance...The more you engage in dynamic reasoning [processes], the less chance there is for dissonance between the old belief and the updated, [improving] belief to develop, the fewer errors you make, but at risk of a new set of cues being neglected."

Karl Weick (personal communication)

Motor cognition is how we understand our movement and how movement helps us understand the events.

\section{The Final Common Pathway of Failing}

Ensuring effective operations in difficult situations continues to bedevil healthcare and organization science. A system appears to be running well, and leaders believe in the capability of their organization. Yet systems continue to fail, and organizations still experience failure. After the accident at the Three Mile Island nuclear power plant, characterized by Charles Perrow (40) as a "normal accident," academicians and organization leaders searched for remedies. One of the early findings was identifying the High-Reliability Organization (HRO) (41-44), with more approaches coming into existence since. However, we have not seemed to have reached a reliable solution for achieving reliability.

To achieve a safe workplace, do not do anything dangerous. An aircraft carrier has the fewest accidents when in port with its planes secured on deck. The focus on danger may be a product of the outsider's frame of reference (45). Operators within the dangerous context recognize the danger, fear the danger, do not show that fear, and continue working (8). Error, as the "experience of failing," is instrumental in achieving safety. Short, negative feedback, sometimes called an error, denotes the boundary of performance, knowledge, and operations. Error corrects heuristic bias (45).

Perhaps the purpose of safety, reliability, and resilience is not to safely work in a dangerous context but to support the extension of the organization into uncertainty, a new environment, unpredictable circumstances, or to penetrate threats.

The safety, reliability, and resilience literature seem to have two components: causality and remedy, with some overlap. Focus on causality resulting from an error, failure, resilience, followership, complexity/chaos, and human factors move the discussion away from a person's experience in the moment of overwhelming events. Focus on remedies such as leadership, team, decision theory, engineered or designed programs moves the remedies away from those intimately involved. It appears we create categories that make sense to those away from events, make decisions that address the situation using logical sequences, study cognitive processes to ensure clear thought and action, treat stress as something lesser people must deal with while fear reactions become invisible. We gloss over the gaps between theory and practice, fear and thought, and abstractions and concreteness.

What makes sense for this approach is using the lexical elements, lexicon, and phrases that develop deep within the experience of those who have worked in a live-or-die context. Failure, then, would not be due to mistranslations but to misapplications by subordinates (2). Reviews then focus on what reviewers, themselves, would have never considered doing rather than how they would act in similar circumstances. This subtle form of blame ("That is something I would never do. Why did they?") is the source of humor for lectures while protecting those outside the event - they now feel safe because they would not have done that.

What is missed with deadly consequences is the action that happens within the funnel of activity that forms from the diverse circumstances that initiate failing, drawing to the singular point of failure. What is missed is the function and utility of error and how failure may be a product of expectations or stopping too soon. Operations within this final common pathway are the same in all situations. What differs between effective operations and ineffective responses is not due to the industry, organization, or type of activity. The differences arise within several domains, which are 
made invisible because of the time compression of local responses and events.

We can describe this final common pathway through engagement $(2,3,8)$, describing four distinct domains (described below), and HRO operating in a topological space. The act of engagement bridges conceptual gaps and extends responsiveness which, in turn, forms prevention and generates resilience. The four domains are sets of properties with functions and characteristics that differ between stable environments (or environments where we can expect stability) and abrupt change or liminal situations. The concepts of HRO effectively operate when used in a manner dependent on how the organization puts them together, rather than operating as discrete, measurable properties. HRO concepts are close to each other in terms of practical relationships rather than distinct and discrete categories or classifications. Such a set of concepts function in a topological, rather than a Euclidean, space. That is, to follow one concept is to travel along a Moebius strip. You end up on the other side.

\section{Extension from Within}

We do not want this topic to become lost in theory or esoterica. We must remain rooted in the concept that we "learn" HRO when we are young and inexperienced. We use the dynamics of HRO throughout our daily routines. Our discussions come from these early experiences that we later apply to extreme circumstances. Experience grows from experience (3). As well, how do we learn from those whose failure turned into wisdom?

Hitting a patch of ice under the snow while descending a mountain one winter, I (DvS) fell quite a distance. Losing my ice axe when the pick caught another patch of ice, I began to spin face down. Not sure how to stop, I began to weigh the advantage of landing at the bottom head first or feet first. A quick death from head trauma or break my thighs and perhaps survive through the night for possible rescue. Then I was not moving. Now I had a different problem. What was holding me in place on the thin snow over scree (small gravel)? It could be one of my knees, my toes, my elbows. With my face pressed into the snow, I thought it could be my chin and nose. This was important to me since I did not want to move my anchor without first creating a better anchor. BTW, I was 18 years old at the time of the fall. The year before, a climber in my climbing group fell to his death in this same way.

Picture cords tied to each wrist. They both pull on you, but you cannot tell which cord is pulling you down the mountain and which is pulling you up. Which cord do you release? That is what happens when the naive enters a dynamic, unstructured state. We observe them holding dearly to beliefs and behaviors learned in a safe, controlled, structured environment, and not only can they not sort out what works from what hurts, they are afraid to let go even if they know.

\section{"That is what happens when the naive enters a dynamic, unstructured state. We observe them holding dearly to beliefs and behaviors learned in a safe, controlled, structured environment, and not only can they not sort out what works from what hurts, they are afraid to let go even if they know."}

People commonly create binary decision frames - let go with the right wrist or the left, go or stay, cut the red wire or the black wire. This may frame the situation for managing risk, but the HRO exists to engage risk. An operator with a Special Operations Group (SOCOMM, Special Operations Command) asked the author (DvS) to explain risk and risk management. "Risk is a determination whether to accept a project. You cannot do that, like I cannot decide to accept a resuscitation. Risk for you is to evaluate your capabilities, augment where you are short, then take the mission." On the mountain, rather than choose a limb to move, the author identified the limb with the most freedom of movement. That is the limb he used to dig in for a stable platform.

In a wildland fire, running from a firestorm, we do not drop our tools. It is not because we are responsible for them or we are captured by fear, but because we were told our tools will save our lives -- until they do not. Smokejumpers encumbered by their tools in the Mann Gulch Fire could not reach safety. Their fire foreman, Wagner Dodge, called out, "Drop your tools!" His crew held onto their tools. Thirteen men died (46). Experiencing a fire burning up a steep slope on a hot day and you are near the ridgeline, does your tool protect you, or does it kill you? Is there a sharp line? Tools for the Neonatologist are protocols and evidence-based medicine.

\section{"Experiencing a fire burning up a steep slope on a hot day and you are near the ridgeline, does your tool protect you, or does it kill you? Is there a sharp line? Tools for the Neonatologist are protocols and evidence-based medicine."}

Teachers, academicians, and consultants from the structured environment are no help. Experienced veterans from the unstructured environment lack the words and defined principles to make sense for the "danger-naive." Early Mount Everest climbers encountered this tension between climber and scientist, experience and model, contextual practice, and decontextualized theory (1, 47-49). Supplemental oxygen may make climbing less difficult but would not help gain altitude. The gap became visible in practice.

Scientists used research from high altitude flights to warn high altitude climbers who use oxygen of sudden unconsciousness from disruption of the oxygen flow (1). Since climbers at the same altitude were conscious, one could surmise two climbers standing together, one with an oxygen supply and one without. Disrupted oxygen flow would cause one climber to lose consciousness while the climber not using oxygen apparatus remained conscious... at the same altitude. Scientists also suffered credibility from their lack of familiarity with the environment. The oxygen apparatus gave the climber a higher center of gravity. Maintaining verticality on a steep slope, the climber had less margin for error before losing balance. A slightly excess lean to either side, and the climber falls to his death. In the liminal environment, inaccurate models can kill (47).

It is counter-intuitive, but what works across the threshold inside the unstructured environment, the liminal space, also works in the structured environment. This is because everyone, from the most naive among us to the most experienced, encounters a situation where they, alone and without support or review, must decide on an action. They feel the knot in the stomach. That is at the thresh- 
old, and those who act find they reduce the discomfort or dissonance by acting while risking visible failure. Others do not act, and their inaction becomes organizational knowledge never to be challenged $(4,50)$.

\section{"These dynamics form the basis of all actions in all industries, organizations, and activities. It occurs from the youngest, least experienced, to the most senior in the organization."}

These dynamics form the basis of all actions in all industries, organizations, and activities. It occurs from the youngest, least experienced, to the most senior in the organization. Bob Bea, Professor Emeritus, Civil Engineering, University of California, Berkeley, maintains a database of over 600 engineering failures and has studied some of the largest engineering disasters in recent US history (51). Bea has also had the experience of a novice placed in a dangerous context.

"When I first went to work offshore - it was as a roughneck handling pipe on an offshore drilling unit. I was given no instructions about what to do or how to do it. But I was told to keep my head out of my XXX, or I would probably lose it (one of my fellow workers later did lose part of his face/head when a drill pipe hit him in the face). Terror is a fine instructor. Later, one of the workers lost three fingers in a cable shiv up on the monkey board in the derrick - 100 feet in the air. He went to the hospital via boat. Guess whom they sent to take his place? Up I went - no instructions - again, terror is a fine instructor."

"Our [dangerous] work has termed this interactive - realtime assessment and management of risks. This approach was completely overlooked until the early 1990 s. We were taught that there was only proactive (before operations) and reactive (after) - and that was it. And we thought we could capture all of the risks with the proactive approaches - and then provide adequate defenses if 'justified' - but we were missing some really major risks that were fundamentally unpredictable and unknowable."

Bob Bea, 8/30/2005, personal communication

We do not think of HRO as a "safety program." Though HRO emerged from dangerous contexts $(8,45)$, "we achieve safety through operations and operations through safety," the mantra of the US Navy and firefighting. Weick captured this in the HRO characteristic "Sensitivity to Operations" (52) when he observed how a disruption distracted an organization leading it to misjudge what was happening (personal communication).

"As a result of my very painful recent experience studying Hurricane Katrina New Orleans, BP Deepwater Horizon, and PG\&E San Bruno disasters, I have come to be very uncomfortable when the word 'safe' is used. In all of these experiences, the people who were 'operating' the systems (at the pointed end of the activity spears) thought they were 'safe enough.' They were not required to 'prove it.' They based their assessments on beliefs and feelings....that proved to be tragically wrong."

Bob Bea, personal communication

Human performance and organizational operations have extended people and organizations into adverse situations or environments. Likewise, they have extended people and organizations into new areas for productivity, in business terms, simultaneous "explore and exploit." HRO describes these organizations. Unfortunately, these descriptions are prescriptive - we know what and why, but not how.

An ecological approach, extending operators into an adverse environment, makes this extension visible into a possible world. High altitude climbers dealt with the primary problems of oxygenation and hypothermia, reliant solely on their attitudes, strength, and materiel readily available. Their methods to solve these problems can inform Neonatologists who also work with problems of oxygenation and hypothermia. While the NICU has great resources readily available, the fragile physiology of the neonate compresses time, effectively creating an austere environment.

The world is a product of study, either as the actual world in which we live or a possible world we strive to inhabit. Neonatology operates in the actual world of infants entering the world before complete physiological development. In the past, these infants would die. Their actual world was a world of the dying. Physicians could only support compassionate death, for example, an infant with hypoplastic left heart syndrome or extremely low birth weight. Conjuring new possible worlds inspired Neonatologists to gain life for these infants (53-56). To make these possible worlds become an actual world for these infants and their families, Neonatologists traversed a liminal environment. Any action could help or hurt, and at any moment, what once hurt will help and what helped could now hurt.

\section{"To make these possible worlds become an actual world for these infants and their families, Neonatologists traversed a liminal environment. Any action could help or hurt, and at any moment, what once hurt will help and what helped could now hurt."}

While operators work to extend operations from within, observers from a fixed point outside of the trajectory of events will share their expertise from that point of view. The British Everest Committee made decisions for climbing Mount Everest, yet none of the members had experience with high altitude climbing. Similarly, within the NICU, infants who would have died will survive with severe disabilities, drawing the attention of outside observers. In their respective liminal environments, high-altitude climbers work to gain altitude, and Neonatologists work to gain life. Outside observers often lack understanding of how one extends operations solely from capabilities and local, immediately available resources. They share the same domains and responses within the final common pathway. These observers missed the possible worlds becoming actual, of dying or disabled infants returned vibrant and thriving by the Neonatologist to their parents. This is the problem HRO endeavors to solve.

Emerging from actual operations within dangerous contexts, HRO extends operations and the organization into uncertain, adverse, and hostile environments. The actual world is an open system where energy, resources, and people are mobile and free to enter and leave. Energy can unexpectedly transform or dissipate through entropy. Novel or uncontrollable situations, uncertainty, and unpredictability constrain human cognitive abilities and thinking. Threats drive behaviors in unexpected ways that seem prudent and logical at the time. HRO describes effective personal and 
organizational characteristics for operations in these liminal environments. Neonatology has much in common with known HROs and can benefit from $\mathrm{HRO}$ as an operational model.

\section{The Four Domains of Engagement}

Introducing residents and staff to critical care while developing a PICU, the author (DvS) (6) presented high-risk operations from four domains. These domains supported the extension of pediatric critical care into critical care transport, EMS, home mechanical ventilation, long-term subacute care, and work with public safety and groups in SOCOM (Special Operations Command). For over 30 years, these have become the most requested domains for lectures and articles, regardless of the discipline or organization.

- categorization

- $\quad$ methods of decision making

- the significance of affective processes

- $\quad$ modulation of the stress and fear responses

The difficulty in translating this method to other industries lies in the interests of corporate administrations for a risk assessment and management model that reduces risk and avoids failure. Business management seeks mastery, accountability, and operational command, particularly for surprise and novel situations. Organizational science builds on models, using quantifiable outcome measures from assessment and transformation tools.

The interests of these HRO "customers" have shifted emphasis away from the characteristics of the liminal environment and the operator's goal of redirecting the trajectory of events from within the event. Safety, reliability, and resilience are becoming consequences of causation, classical predicate (scientific) logic, categorization, and the cognitive sciences. Human intentions and organizing structures and systems from outside, a safer place to be, now inform design and engineering. These new dominant accounts have washed out the strength and meaning of HRO.

\section{"HROs focus on function and consequences. As Weick observed (personal communication), a disruption can distract an organization leading it to misjudge what is happening."}

HROs focus on function and consequences. As Weick observed (personal communication), a disruption can distract an organization leading it to misjudge what is happening. While collecting sufficient information to act, circumstances have changed -- when you do not have the necessary information, acting immediately. This is the uncertainty principle bedeviling all who arrive at an exigent situation. HROs focus on function. With insufficient information as a given, the HRO generates information through action.

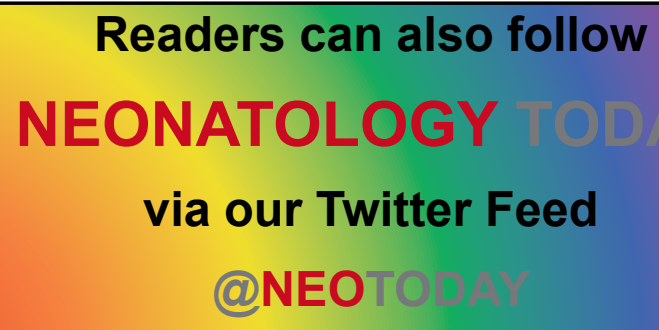

@NEO
HROs focus on consequences.

The organization must extend its operations into new environments, sustain activity, and transform this actual world into a new, better world. The HRO stands up and changes the world while the world changes the HRO - both for the better. Strength through change - allostasis. That is the goal of extension.

For this to happen, members of the HRO must think clearly while in adverse situations. They suppress their fear reactions (57) and modulate their stress and threat responses (22) with flexibility from the affective processes (6). Decision-making is a continuous, adaptive process focused on negative feedback to ensure safety (58). HROs categorize their actions, flexible thinking, and options for function, salience, and relevance.

\section{"High-Reliability Organizing, the verb form (10), maintains active forward motion from its topological geometry of adaptive relationships compared to Euclidean programs of points and connections."}

\section{Categories}

The author (DvS) was involved with several types of medical situations in the same category:

- Physicians on a state EMS committee argue about the minimum blood pressure for the administration of nitroglycerin by paramedics. The author realized the physicians from cooler, northern regions wanted the lower blood pressure used while those from hotter arid regions wanted the higher blood pressure.

- A pediatric emergency medicine fellow has administered $40 \mathrm{~mL} / \mathrm{kg}$ saline to an infant with diarrhea. Her attending tells her that, after administering half a blood volume of fluid, she should start dopamine for heart failure and transfer the infant to the PICU. She gives another $30 \mathrm{~mL} /$ $\mathrm{kg}$ and transfers the infant to the ward.

- The PICU transport team treats a two-week-old infant in the ED with intubation and $100 \%$ oxygen. The heart rate remains $100 \%$ and oxygen saturation about $70 \%$. They call the attending for recommendations to increase oxygen saturation.

- A paramedic in a southern state responds to an overturned vehicle hidden down an embankment. It has been hot and humid for several days and nights. An elderly lady in the car, missing overnight, has heart medication in her possession. He follows state protocol and treats her for heart failure by administering dopamine. She does not respond. Following protocol, he increases the rate of infusion. She dies on the way to the hospital.

- A physician in an arid region had been administering fluids to the elderly who are in heart failure. He had just lost his staff privileges for providing significantly lower than the standard of care to treat heart failure.

These patients fit the category of heart failure. Though in the correct category, they were also in the category "hypovolemia" and "dehydration." Clinically distinguishing between heart failure with normal blood volume and heart failure with low blood volume is 
difficult. The two treatments conflict -- fluid restriction for heart failure and fluid administration for hypovolemia. That is likely why no one considered these situations to be errors. One individual understood the management for this physiological conflict. He lost his job.

There are various ways to create categories. Time and space create sequential and parallel categories, respectively. Laws, rules, and logic drive categories by influences external to the system. Categories can preserve the structure, mapping elements in a Euclidean space while others group by sets and relations. Not as familiar in healthcare, topological categories group manifolds (forms), and their evolution is passing to the continuum (59). This categorization may become useful for studying organizational disruptions and $\mathrm{HRO}$ as happenings in a topologic space.

\section{"In Kantian philosophy, all elements can be contained within known concepts, with a fundamental principle being causation. Refined since Kant's original work, concepts have become a foundation of modern science (3)." "}

In Kantian philosophy, all elements can be contained within known concepts, with a fundamental principle being causation. Refined since Kant's original work, concepts have become a foundation of modern science (3). The Linnean system categorizes typologies into taxonomic hierarchies to show relationships. ICD-10 codes create discrete categories out of continuous processes for public health data analysis. The categories of evidence-based medicine are intended to reduce waste and medical complications.

Less formally, we categorize using our own experience as our frame of reference. We translate the situation with those terms we normally use. If we only have words and categories for aggressive behavior, often learned from television and movies, then we use them to describe a confused and agitated parent. When we do not have the right category, we find one we think fits.

For classification systems, a derivative of categorization, ambiguity in classifying can balance service to multiple constituencies. Others may be excluded; the 'other' category, called "residual categories" by Leigh Star (60), can render elements and people invisible. Such people in the organization's operations may have acquired valuable experience and can offer useful insights during severe liminal episodes (61).

"Boundary objects" are ambiguous yet constant objects shared by adjacent categories. This allows their use by several communities of practice, such as meeting their educational requirements (62). Boundary objects allow for cooperation and communication across borders, often without the need for different groups to communicate, and can help manage the tension between divergent viewpoints (61).

HROs work in liminal environments with ill-structured problems embedded in unstable environments. Standards, residual categories, and boundary objects for an HRO are fluid. Over time, leaders, administrators, and regulators will mistranslate the nature of an HRO and try to control or reduce this fluidity (60). This is usually accomplished with increased standardization and reduced diversity and variability.

\section{Decision making}

In the liminal zone, decisions become a process over time with se- rial evaluations and corrections. In motor cognition, the intention is not complete until the motor function ends (34). Because we do not know the situation, our objective guides decision-making. We have several simultaneous objectives since we may not reach our desired objective-we direct efforts to increase the chance of success while decreasing failure. If we cannot reach an objective, we decompose it until we begin moving toward our objective (6).

"How do you manage error?" A federal health agency posed the question at a national conference for $\mathrm{HRO}$ in healthcare. There was silence at the author's (DvS) table. The author then stated that an error is golden. "It tells me where we are weak, what to teach, what people don't know, where the problems are... error is valuable" The CEO of a major healthcare system, proud of their HRO program, sat in silence. For the remaining 10 minutes, no one spoke.

For the ill-defined, embedded problem, we rely on heuristics for shortcuts, constantly re-evaluate, vigilant for errors. Error corrects heuristic bias. Error marks the limits of our individual and team performance and the organization's capabilities. That is how error makes us safe (58).

For the novice, we gave two guidelines in these situations (6).

- Is it reversible? If so, it becomes a titration. If not, consult with others.

- What would happen if you treated too soon or too late? What would happen if you gave too much or too little? This creates a decision frame the neophyte can operate within.

Fundamental differences for decision-making in liminal environments and stable environments lie in the purpose and function. Decisions and actions have the purpose of generating information. Even unwanted results are information with meaning and relevance. Error for outsiders is unwanted, sometimes worthy of opprobrium. Error then creates the ecology of fear $(58,63)$. For the $\mathrm{HRO}$, "error" has functions to identify boundaries of performance and operations, the "envelope" that HROs seek to expand. "Error" identifies a mismatch between what is known and what is uncertain or ambiguous. "Error" identifies unexpected or unrecognized changes in conditions or the environment.

The authors, coming from operational environments, had not encountered the everyday use of error for operations. This makes sense because, in the operational environment, we do not know what works until we act. One cannot find solutions by reasoning. One cannot organize or build a structure from the outside. We must enter the problem, thus becoming part of the problem (2).

\section{Affective processes}

Neonatologists are often more comfortable with cognitive processes - objective, reasoned, and classical predicate (scientific) logic. Most of all, cognitive processes readily incorporate evidence-based medicine. However, in the flux of changing events and unrecognized fear, the Neonatologist must give value, meaning, and relevance to changing information in unstable contexts.

To support the function of HRO in unstable circumstances, we have characterized the affective processes or domain (6):

- $\quad$ attitudes and values

- $\quad$ situational value of information

- neuromodulated emotion

- the Affective Domain of Learning (Bloom's Taxonomy) (64)

Attitudes and values 
Attitudes influence and follow behavior. "Attitudes are preferences, 'the predisposition to treat entities with favor or disfavor'” (65). Attitudes drive action and influence the meaning we give to uncertain or ambiguous information.

The five HRO principles (52) describe natural, effective, adaptive responses to adversity and hostile environments. Positioned as attitudes, we see how they develop from experience and frame the processes necessary to reach High-Reliability Organizing:

- $\quad$ one's attitude toward failure

- $\quad$ acceptance of the complexity of even simple events

- $\quad$ awareness of how one fits into the scheme of life (situation-centric versus person-centric)

- perseverance

- $\quad$ respect for the knowledge and experience of others approaching an emergency.

The meaning of information changes during events and also among participants. There is no useless information in an emergency.

The relevance of information, how you use it, is also situational.

The value of information, the sum of the above, shifts and changes throughout the event.

Neuromodulated emotion. See below.

Affective Domain of Learning (Bloom's Taxonomy),

All the time, effort, and cost of teaching can be lost in seconds. "I know that's what they taught you. Let me tell you how it's really done." Teaching from Bloom's Affective Domain of Learning brings focus to "How this will help." That can become a mantra of bedside teaching - how this will help you treat the baby and support the parents.

People respond to the environment in ways that make sense to them. Teaching from the Affective Domain helps students make sense of the environment and the situation. This is also contextual teaching.

\section{"The affective domain of learning includes awareness, responsiveness, attitudes, placing value on information, and internalizing values."}

Values are more strongly held and more permanent beliefs compared to attitudes. We use our values to judge information and actions. Though values are personal, some specific values are binding us to our cultures. In the HRO, some values shift as needed, such as obedience and conformity in low tempo times shift to initiative and creativity in high tempo events. We have identified five values of HROs (6):

- Duty. We will not let others down; we have a duty to our larger community

o "Responsibility" or "accountability" can be limiting

- Empathy. We are all capable of making mistakes at virtually any time; HROs work in tough situations where people are going to fail

o "Compassion" will focus on the other person or response toward that person

- Dignity. Acknowledge the value of everybody's contribution, every job, every task, is important

o Discipline people without taking away their dignity

o "Respect" is generally considered earned and can easily be lost

- Honesty What you say represents the circumstances

o "Trust," like respect, is transactional between two people

- Humility. The foundation of learning, required to improve people

Situational value of information.

The salience of signs and information prevents distraction when
The affective domain of learning includes awareness, responsiveness, attitudes, placing value on information, and internalizing values. We provide the context necessary to give meaning to information. This increases motivation to learn and to understand better the knowledge we have gained. Motivation, as affective learning, describes responsiveness to phenomena, the valuing of knowledge, and the worth applied to an object or idea.

Effect on decision-making. See above.

\section{Stress and fear responses}

The negative results from unmodulated stress, fear, and threat distract us from their adaptive and survival functions. The overlap of their effects confounds our ability to reach practical management approaches. They each respond to different signals, develop in different brain regions, and have different purposes. Organisms do not have a panoply of responses nor the ability to select the response measured to the attack. This all-or-none system makes sense because, in the beginning, the most severe attack looks no different than a mild attack. Only the time course differs $(22,66)$.

Stress responses are from the amygdala, and the neurochemical (cortisol) response to novelty, uncertainty, and uncontrollability. Impairment of the prefrontal cortex constrains executive functions and abstract thought. Impairment of the hippocampus blocks memory retrieval except for procedural (habit or motor) memory, which is enhanced. The effect of intentional motor activity on thought as motor cognition, described above, may explain why intentional movement can break the grip of cortisol on thinking $(22,66)$.

Fear maintains a safe distance from the "enemy" by the "escape reaction" to maintain a "flight distance" (67). Enemy proximity (it need not be a predator or other threat) is initially processed in the ventromedial prefrontal cortex (vmPFC) but moves to the midbrain periaqueductal gray (PAG) nucleus to coordinate survival 
behaviors. In physical settings, the organism moves to maintain the distance ("fear flight"), and fighting is for personal protection or escape ("fear fight"). In psychological settings, the individual adopts defensive protection ("fear flight") by avoiding what the person believes is the problem, using rationalizations and abstractions, and deflecting, excusing, or justifying. For offensive protection ("fear fight"), the individual acts verbally against others with surprise, concentrated action, fast tempo, audacity, blame, accusation, and personal attacks $(22,66)$.

\section{"We can, however, learn to neuromodulate the behaviors in ourselves or assist and support others to recognize and neuromodulate their threat reflexes $(8,70) . "$}

The threat is a subcortical reaction to existential danger. Known for the "fight or flight" reflexes $(68,69)$, other threat reflexes tonic immobility, and attentive freeze. As it is Initiated through the amygdala, the threat is a bona fide reflex as we cannot prevent the initiation of either the affective or motor component (68). We can, however, learn to neuromodulate the behaviors in ourselves or assist and support others to recognize and neuromodulate their threat reflexes $(8,70)$. Managing threat reflexes are discussed elsewhere $(22,66)$, but they manifest as:

- 'Fight - anger or frustration

- $\quad$ Flight - avoidance or distraction (searching for equipment is the motor component absent the affective component)

- $\quad$ Tonic immobility - prevents physical movement despite awareness; milder presentations are intense aversion, gastric upset, or nausea

- $\quad$ Attentive freeze - physical freeze with full awareness, the freeze is in the motor component; cognitive freeze with physical activity, the freeze is cognitive from the affective component

The greatest significance of the threat reflexes is their mundane appearance - sick to the stomach and avoiding a decision, anger directed at staff or the situation, diversions for more information, or inability to recall well-known information, as a few examples. In the authors' experience, the knowledge that these behaviors and cognitions are neurochemically mediated helps in the prevention, management, or termination of the behaviors.

People working in dangerous contexts neuromodulate these responses $(8,22)$. As HRO has entered healthcare and business management, this seminal characteristic of high-reliability operations has been missed $(2,45)$.

\section{Inhibitions of Extension}

The extension of neonatology asserted effective care over ever more difficult physiology in the liminal zone surrounding a dying baby. Babies and their families benefitted. Extension of care, like $\mathrm{HRO}$, is an ongoing process with new people, disease-physiology interactions, and new families. The organization uses the same HRO methods to achieve, sustain, and extend HRO operations.

Engineered designing to achieve safety, reliability, and resilience for technological systems or socio-technical systems is seductive. It appears clean and capable of preventing or mitigating consequential bad outcomes. The weakness is not compliance from staff. The weakness is from the experience of individual staff facing a confusing situation. They will always act in a way that makes sense to them...locally. This may not make sense to a spectator (2).
"Technological systems become organized by commands from the outside, as when human intentions lead to the building of structures or machines. But many natural sys- tems become structured by their own internal processes: these are self-organizing systems, and the emergence of order within them is a complex phenomenon that in- trigues scientists from all disciplines."

\section{Eugene F. Yates (71)}

Socio-Technical Systems (STS). Adding social structure to a technological system, a form of STS does not solve the problem. STS was first described in British underground coal mining with the introduction of technology to mine long-wall segments at one time, by the new "long-wall" method. Until introducing the new technology, lifetime teams of two men worked face-to-face with the "room-and-pillar" technique, which provided social balance. Some mines showed increased productivity with greater cohesiveness and personal along with decreased sickness and absenteeism. Other mines, however, did not. The difference was how the technical management gave miners the security necessary for undertaking new developments (72).

The authors evaluated the outcomes by regarding the long-wall as a technological system expressive of engineering interacting with the long-wall regarded as a social structure with occupational roles. Lost from the room-and-pillar technique was adaptability and responsible autonomy. Mechanization brought new problems:

- intermediate organization from direct face-to-face work

- the lack of recognition of the nature of difficulties

- $\quad$ segmented quality of social organization from a change in scale and spatio-temporal structure

\section{"Disregarded were the dangers and} the human shortcomings that followed, the vulnerability of the socially isolated individual, and the magnification of local disturbances."

Disregarded were the dangers and the human shortcomings that followed, the vulnerability of the socially isolated individual, and the magnification of local disturbances. While leadership "organized by commands from the outside," the miner's social "systems become structured by their internal processes." The resentment and hostility of the miners self-organized for group defense. Technology can create a socially ineffective structure. Yet good management and leadership with close interaction with the staff do produce far better results. We can treat technology as two systems, a technological system, and a social system. HRO works

\section{Readers can also follow} NEONATOLOGY TODAY via our Twitter Feed @NEOTODAY 
like that, observed in the organization of the USS Carl Vinson, the aircraft carrier studied by the University of California, Berkeley, HRO study group $(41,45)$.

Fear. Implementation of safety and reliability appears more straightforward with structure, error prevention, and standardization. Other methods address the concern about regulatory or accreditation violations, liability concerns, or financial challenges. The indirect effect is to create situations where damage to the organization from the fear of error or violation is greater than the actual threat. We enact our operations and restraints. Failure from not acting is invisible and becomes part of the organization's knowledge (50). This is the invisible ecology of fear (73). Reinforcing this fear are the stories told to novices to engender fear and not for instruction, the pedagogy of fear (74).

Quality improvement (QI) auditors create their own type of fear. A former paramedic colleague of the author (DvS) requested a transfer to the EMS QI section of the fire department. His colleagues warned him; the program had a poor reputation. Discussing with the author whether to accept the assignment, the author described Philip Zimbardo's Prisoner-Guard Study (75). The QI auditors are guards, protecting the department from the paramedics, who became the prisoners. To prevent this from happening, he charged the auditors with the task of helping and supporting paramedics. After one year, the members of EMS and the fire department thought highly of the EMS QI section.

People do not self-organize for extension into uncertainty if they work or live in the ecology of fear.

Disrupting engagement. Decision-making for the indeterminate problem described above starts with the objective, for example, the airway in the resuscitation ABCs. We should not sacrifice the time necessary to move toward safety to know where we are (soon to be "were") or identifying antecedent events. Bea describes this approach (above) as interactive - real-time assessment and management of risks. Two unrecognized disruptions to engagement are the category for outliers and the rigor for following rules and protocols. The extension follows engagement. Disrupted engagement means lost opportunity and blocked the extension of neonatology care.

An outlier, to the healthcare practitioner steeped in science, is several standard deviations from the mean. In healthcare, we use statistics, a measure of the past, and probability, a projection into the future. Random and independent, not connected to the present, not useful for the future, we readily disregard the outlier. (Think zebras, a quite dangerous, aggressive equine.) "The key problem in statistics is the validity of the data that has been gathered. The key problem in probability is the potential for changes in conditions in the future that invalidate the data that has been gathered during the past," Bob Bea. Bea had a warning about disregarding outliers:

"That depends on the outliers. This 'aliasing' data got NASA into deep trouble with the 'O-rings' in low-temperature launches that became apparent during the Challenger disaster. The 'outliers' were data from a 'different population' (low temperatures that could change the properties of the O-rings...making them brittle). The 'parent population' was for higher temperatures that did not result in the embrittlement of the O-rings. Outliers must be carefully treated because they can really hurt...or help you."

\section{Bob Bea (personal communication)}

The expert following the rules. An organization or industry may institutionalize competency rather than proficiency because generalizable principles can be taught to a high-turnover, novice workforce (76). Moving to proficiency requires the use of specifics, the particular, and case reports. It demands more time, training, shared insight, and modeling of senior, more experienced people. Competency-based programs form the rule-based system of Jens Rasmussen's skills, rules, knowledge (SRK) framework in his ecological interface design system (77). James Reason (78) adapted this framework for his model of error management now used in healthcare. This is almost institutionalization to impair institutional extension.

\section{"Experts continue to develop, and people gain mastery of concepts despite this focus on rule-based systems and rule- based error."}

Experts continue to develop, and people gain mastery of concepts despite this focus on rule-based systems and rule-based error. Though we may master concepts, we never master experience (3). Rasmussen recognized this. Local or special knowledge is the knowledge-based system. in extreme cases where the particular is novel or unexpected

"Operators are maintained in [complex technological] systems because they are flexible, can learn and do adapt to the peculiarities of the system, and thus they are expected to plug the holes in the designer's imagination."

Jens Rasmussen (79)

This is how the organization supports extension into novel or adverse environments. But we can now see the problem. An institutionalized rule-based system, developed for the workforce enacted by the organization (competency-based restraint), and an organization desiring extension into a knowledge-based environment must follow the rules in a knowledge-based situation.

We process the familiar on the right side of the brain and the novel on the left side. The Dreyfus brothers knew this when they studied

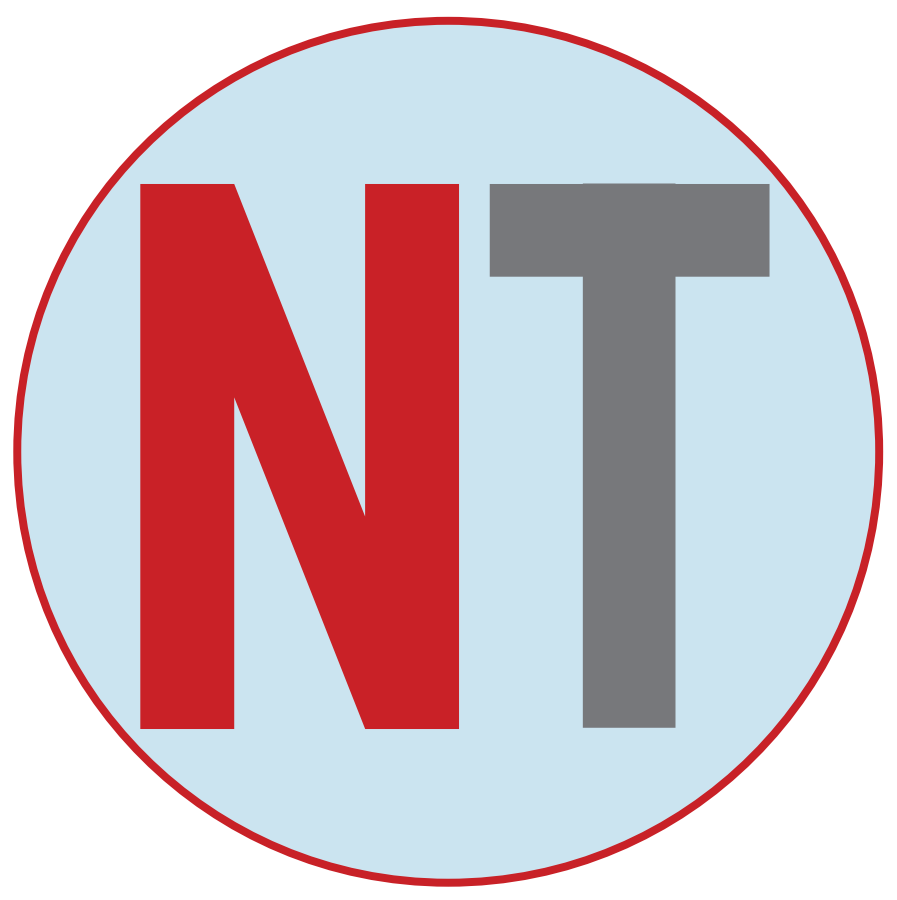


the expert following the rules $(80,81)$ "True mastery comes when the performer is conscious neither of identifying the situation nor of remembering an action, but rather act spontaneously without necessarily becoming consciously aware of his situation" (81). When the expert operating in this manner, yet required to follow a rule or protocol, will encounter an inappropriate set. This shifts the manner of thought and brain regions. Performance suffers. The expert operates at a lower level than the novice. What has not been explored is motor cognition and the consequences of an expert constrained by the rules.

The organization inhibits initiative. Correlation or association does not mean causation. We have heard this statistical observation used to end a discussion. There are several other forms, "Such results suggest that an error has been made, of an old kind, in arguing from correlation to causation; we cannot argue from Correlation to causation" (82). "The association observable between the practice of cigarette smoking and the incidence of cancer of the lung...has been interpreted... as though it demonstrated a causal connexion between these variables" and "Dr. Bradford Hill while admitting that the evidence of association found by his Unit did not amount to proof of causation" (83). These cited quotations are from Ronald A. Fisher, an inveterate pipe-smoker developer of the Analysis of variance (ANOVA), introduced the null hypothesis, proposed the level of $p=0.05$, and developed modern experimental design. In dangerous contexts, sometimes association or correlation is all you have.

"Correlation does not mean causation" is a "thought-terminating cliché," a type of phrase used to block further analytical thinking. Once someone uses such a phrase, no further argumentation is required by the speaker and no argumentation is allowed by others. It is a form of "loading the language" used during indoctrination processes. Complex problems are compressed into brief, definitive-sounding phrases that can be easily memorized and easily expressed. To others, these phrases cause constriction and linguistic deprivation (84).

To block inquiry and investigation is to impair extension.

\section{What it means to be a Neonatologist}

We don't find meaning; we give meaning. For example, list what you do as a Neonatologist. Include tasks and activities.

Adding to the list becomes more difficult as the list grows. When your list has at least seven items, preferably ten, remove the first 3-4 items. The things you think of first are the easiest to think of, not the most important.

The last things on your list...those that are the most difficult to conjure, are what it means to be a Neonatologist. This is where you give meaning to your work. That meaning increases your stress capacity. This is the affective domain.

These are also the items that drive the extension of neonatology. They are how neonatology will extend into new worlds and how the Neonatologist gains life.

When a person believes in themselves, they will leave their place of safety and engage the unknown when they know they are supported. Changing a deteriorating problem for the better, knowing their sense of agency helped a struggling baby, seeing life improve...that is where resilience comes from. That is HRO. That is how we extend the care of the Neonatologist to babies and their families. That is how the Neonatologist and the discipline of neonatology gain life.

A tremendous amount of time, effort, and cost are expended teaching healthcare professionals how to develop and ensure reliability, safety, and resilience.

We know that is what they taught you. Let us tell you how it is re- ally done.

\section{References:}

1. van Stralen D, Mercer TA. High Altitude Climbing, High Reliability, COVID-19, and the Power of Observation. Neonatology Today. 2021;16(1):68-79. doi: 10.51362/neonatology. today/20211616879.

2. van Stralen D. Pragmatic High-Reliability Organization (HRO) During Pandemic COVID-19. Neonatology Today. 2020;15(4):3-9.

3. van Stralen $D$, Mercer TA. The Nature of Neonatal Experience during Pandemic COVID-19. Neonatology Today. 2021;16(3):87-97. doi: 10.51362/neonatology.today/202131638797.

4. Weick KE. Enacted sensemaking in crisis situations Journal of management studies. 1988;25(4):305-17.

5. Weick KE. Enactment processes in organizations. In: Straw $B$, Salanick $G$, editors. New directions in organizational behaviour. Chicago, IL: St. Clair; 1977. p. 267-300.

6. Van Stralen D, Inozu B, Byrum S. High Reliability for a Highly Unreliable World: Preparing for Code Blue through Daily Operations in Healthcare. North Charleston, SC: CreatSpace Publishing; 2017.

7. van Stralen D, Mercer TA. The Art of Neonatology, the Art of High Reliability as a Response to COVID-19. Neonatology Today. 2021;16(2):74-83. doi: 10.51362/neonatology. today/202121627483.

8. van Stralen D, Mercer TA. High-Reliability Organizing (HRO) in the COVID-19 Liminal Zone: Characteristics of Workers and Local Leaders. Neonatology Today. 2021;16(4):90-101. doi: 10.51362/neonatology.today/2021416490101.

9. Robichaud D, Giroux H, Taylor JR. The Metaconversation: The Recursive Property of Language as a Key to Organizing. Academy of Management Review. 2004;29(4):617-34. doi: 10.5465/amr.2004.14497614.

10. Weick KE. The social psychology of organizing. 2 ed. Reading, MA: Addison- Wesley; 1979.

11. Weick KE. Reflections: Change Agents As Change Poets - On Reconnecting Flux and Hunches. Journal of Change Management. 2011;11(1):7-20. doi: 10.1080/14697017.2011.548937.

12. Whitehead AN. Science and the modern world. Cambridge, UK: Cambridge University Press; 1926.

13. James $W$. The principles of psychology. New York, NY: Dover; 1890 (1950).

14. Jeannerod M. Motor cognition: What actions tell the self. Oxford, UK: Oxford University Press; 2006.

15. Chambon V, Haggard P. Premotor or Ideomotor: How Does the Experience of Action Come About? In: Prinz W, Beisert $M$, Herwig $A$, editors. Action science: Foundations of an emerging discipline. Cambridge, MA: MIT Press; 2013. $p$. 359-80.

16. Weick KE, Roberts KH. Collective mind in organizations: Heedful interrelating on flight decks. Administrative science quarterly. 1993;38:357-81.

17. Rizzolatti G, Craighero L. The mirror-neuron system. Annu Rev Neurosci. 2004;27:169-92. Epub 2004/06/26. doi: 10.1146/annurev.neuro.27.070203.144230. PubMed PMID: 15217330.

18. lacoboni M. Neural mechanisms of imitation. Curr Opin Neurobiol. 2005;15(6):632-7. Epub 2005/11/08. doi: 10.1016/j. conb. 2005.10.010. PubMed PMID: 16271461.

19. Jacob $P$, Jeannerod $M$. The motor theory of social cognition: a critique. Trends Cogn Sci. 2005;9(1):21-5. Epub 2005/01/11. doi: 10.1016/j.tics.2004.11.003. PubMed PMID: 15639437.

20. Sakreida K, Scorolli C, Menz MM, Heim S, Borghi AM, Binkofski F. Are abstract action words embodied? An fMRI inves- 
tigation at the interface between language and motor cognition. Front Hum Neurosci. 2013;7:125. Epub 2013/04/12. doi: 10.3389/fnhum.2013.00125. PubMed PMID: 23576972; PubMed Central PMCID: PMCPMC3620530.

21. Harpaintner $M$, Sim EJ, Trumpp NM, Ulrich $M$, Kiefer $M$. The grounding of abstract concepts in the motor and visual system: An fMRI study. Cortex. 2020;124:1-22. Epub 2019/12/11. doi: 10.1016/j.cortex.2019.10.014. PubMed PMID: 31821905.

22. van Stralen D, Mercer TA. Pragmatic High-Reliability Organizations (HRO) Modulate the Functions of Stress and Fear Behaviors During Pandemic COVID-19: The Stress-FearThreat Cascade. Neonatology Today. 2020;15(10):126-34. doi: 10.51362/neonatology.today/2020101510126134.

23. Boulenger V, Roy AC, Paulignan Y, Deprez V, Jeannerod M, Nazir TA. Cross-talk between language processes and overt motor behavior in the first 200 msec of processing. J Cogn Neurosci. 2006;18(10):1607-15. Epub 2006/10/04. doi: 10.1162/jocn.2006.18.10.1607. PubMed PMID: 17014366.

24. Pulvermuller F, Hauk O, Nikulin VV, IImoniemi RJ. Functional links between motor and language systems. Eur J Neurosci. 2005;21(3):793-7. Epub 2005/03/01. doi: 10.1111/j.14609568.2005.03900.x. PubMed PMID: 15733097.

25. Buccino G, Riggio L, Melli G, Binkofski F, Gallese V, Rizzolatti $G$. Listening to action-related sentences modulates the activity of the motor system: a combined TMS and behavioral study. Brain Res Cogn Brain Res. 2005;24(3):355-63. Epub 2005/08/16. doi: 10.1016/j.cogbrainres.2005.02.020. PubMed PMID: 16099349.

26. Tettamanti M, Buccino G, Saccuman MC, Gallese V, Danna $M$, Scifo $P$, et al. Listening to action-related sentences activates fronto-parietal motor circuits. J Cogn Neurosci. 2005;17(2):273-81. Epub 2005/04/07. doi: 10.1162/0898929053124965. PubMed PMID: 15811239.

27. Anderson MH. How Can We Know What We Think Until We See What We Said?: A Citation and Citation Context Analysis of Karl Weick's The Social Psychology of Organizing. Organization Studies. 2016;27(11):1675-92. doi: 10.1177/0170840606068346.

28. Caligiore D, Pezzulo G, Baldassarre G, Bostan AC, Strick PL, Doya K, et al. Consensus Paper: Towards a SystemsLevel View of Cerebellar Function: the Interplay Between Cerebellum, Basal Ganglia, and Cortex. Cerebellum. 2017;16(1):203-29. Epub 2016/02/14. doi: 10.1007/s12311016-0763-3. PubMed PMID: 26873754; PubMed Central PMCID: PMCPMC5243918.

29. Schmahmann JD. The cerebellum and cognition. Neurosci Lett. 2019;688:62-75. Epub 2018/07/13. doi: 10.1016/j.neulet.2018.07.005. PubMed PMID: 29997061.

30. Gallese V, Rochat M, Cossu G, Sinigaglia C. Motor cognition and its role in the phylogeny and ontogeny of action understanding. Dev Psychol. 2009;45(1):103-13. Epub 2009/02/13. doi: 10.1037/a0014436. PubMed PMID: 19209994.

31. Jeannerod M. Neural simulation of action: a unifying mechanism for motor cognition. Neuroimage. 2001;14(1 Pt 2):S103-9. Epub 2001/05/25. doi: 10.1006/nimg.2001.0832. PubMed PMID: 11373140.

32. Klüver J, Schmidt J. Topology, Metric and Dynamics of Social Systems. Journal of Artificial Societies and Social Simulation. 1999;2(3):7.

33. Mol A, Law J. Regions, networks and fluids: Anaemia and social topology. Soc Stud Sci. 1994;24(4):641-71. Epub 1994/11/01. doi: 10.1177/030631279402400402. PubMed PMID: 11639423.

34. Haggard P. Conscious intention and motor cognition. Trends Cogn Sci. 2005;9(6):290-5. Epub 2005/06/01. doi: 10.1016/j. tics.2005.04.012. PubMed PMID: 15925808.
35. Mylopoulos M, Pacherie E. Intentions and Motor Representations: the Interface Challenge. Review of Philosophy and Psychology. 2016;8(2):317-36. doi: 10.1007/s13164-0160311-6.

36. Pezzulo G, Cisek P. Navigating the Affordance Landscape: Feedback Control as a Process Model of Behavior and Cognition. Trends Cogn Sci. 2016;20(6):414-24. Epub 2016/04/28. doi: 10.1016/j.tics.2016.03.013. PubMed PMID: 27118642.

37. Weiskrantz L, Elliott J, Darlington C. Preliminary observations on tickling oneself. Nature. 1971;230(5296):598-9.

38. Sokolov AA, Miall RC, Ivry RB. The Cerebellum: Adaptive Prediction for Movement and Cognition. Trends Cogn Sci. 2017;21(5):313-32. Epub 2017/04/08. doi: 10.1016/j. tics.2017.02.005. PubMed PMID: 28385461; PubMed Central PMCID: PMCPMC5477675.

39. Fuentes CT, Bastian AJ. 'Motor cognition' - what is it and is the cerebellum involved? Cerebellum. 2007;6(3):2326. Epub 2007/09/06. doi: 10.1080/14734220701329268. PubMed PMID: 17786819; PubMed Central PMCID: PMCPMC4096570.

40. Perrow C. Normal Accident at Three Mile Island. Society 1981;18(5):17-26.

41. Bourrier M. The Legacy of the High Reliability Organization Project. Journal of Contingencies and Crisis Management. 2011;19(1):9-13. doi: 10.1111/j.1468-5973.2010.00628.x.

42. Roberts $\mathrm{KH}$, Bea R. Must accidents happen? Lessons from high-reliability organizations. Academy of Management Perspectives. 2001;15(3):70-8. doi: 10.5465/ ame.2001.5229613.

43. LaPorte TR. On vectors and retrospection: Reflections on understanding public organizations. 2011;19(1):59-64.

44. Rochlin GI. How to hunt a very reliable organization. Journal of contingencies and crisis management. 2011;19(1):14-20.

45. van Stralen D, McKay S, Mercer T. Flight Decks and Isolettes: High-Reliability Organizing (HRO) as Pragmatic Leadership Principles during Pandemic COVID-19. Neonatology Today. 2020;15(7):113-21. doi: 10.51362/neonatology.today/20207157113121.

46. Weick KE. The collapse of sensemaking in organizations: The Mann Gulch disaster. Administrative science quarterly. 1993;38(4):628-52.

47. Heggie V. Experimental physiology, Everest and oxygen: from the ghastly kitchens to the gasping lung. The British Journal for the History of Science. 2012;46(1):123-47. doi: 10.1017/s0007087412000775.

48. Tuckey HP. Everest-The First Ascent: The untold story of Griffith Pugh, the man who made it possible. New York, NY: Random House; 2013.

49. Tilman H. Everest 1938: Whether these mountains are climbed or not, smaller expeditions are a step in the right direction. Cambridge, UK: Cambridge University Press; 1948.

50. Weick KE. Enactment and Organizing. The Social Psychology of Organizing. Second ed. New York, NY: McGraw-Hill, Inc.; 1979. p. 147-69.

51. Prud'homme A. Bob Bea, the Master of Disaster. Men's Journal 2013:72-5.

52. Weick KE, Sutcliffe KM. Managing the Unexpected: Assuring High Performance in an Age of Complexity. Quinn RE, editor. San Francisco, CA: Jossey-Bass; 2001.

53. Johnson JD, Malachowski NC, Grobstein R, Welsh D, Daily WJR, Sunshine P. Prognosis of children surviving with the aid of mechanical ventilation in the newborn period. The Journal of Pediatrics. 1974;84(2):272-6. doi: 10.1016/s00223476(74)80622-0.

54. Adamson TM, Collins LM, Dehan M, Hawker JM, Reynolds EOR, Strang LB. Mechanical Ventilation in Newborn Infants with Respiratory Failure. The Lancet. 1968;292(7562):227- 
31. doi: 10.1016/s0140-6736(68)92348-9.

55. Glover WJ. Mechanical ventilation in respiratory insufficiency in infants. Proceedings of the Royal Society of Medicine. 1965;58(11 Part 1): 902-4.

56. Reynolds EOR. Indications for mechanical ventilation in infants with hyaline membrane disease. Pediatrics. 1970;46(2):193-202.

57. Gioia DA. On Weick: An Appreciation. Organization Studies. 2016;27(11):1709-21. doi: 10.1177/0170840606068349.

58. van Stralen D, Gambino W. Error as a Faulty Failure Signal. Neonatology Today. 2020;15(9):114-7. doi: 10.51362/neonatology.today/20209159114117.

59. Coecke B, Paquette EO. Categories for the practising physicist. In: Coecke B, editor. New structures for physics. 813. Oxford, UK: Springer; 2011. p. 173-286.

60. Star LS. This is Not a Boundary Object: Reflections on the Origin of a Concept. Science, Technology, \& Human Values. 2010:35(5):601-17. doi: 10.1177/0162243910377624.

61. Bowker GC, Star SL. Sorting things out: Classification and its consequences. Cambridge, MA: MIT press; 1999.

62. Star SL, Griesemer JR. Institutional Ecology, 'Translations' and Boundary Objects: Amateurs and Professionals in Berkeley's Museum of Vertebrate Zoology, 1907-39. Social studies of science 1989;19(3):387-420.

63. van Stralen D, Mercer TA. Pandemic COVID-19, the HighReliability Organization (HRO), and the Ecology of Fear. Neonatology Today. 2020;15(12):129-38. doi: 10.51362/ neonatology.today/2020121512129138.

64. Krathwohl DR, Bloom BS, Masia B. Taxonomy of Educational Objectives Handbook II: Affective domain. New York, NY: David McKay Company; 1964.

65. Banaji MR, Heiphetz L. Attitudes. In: Fiske ST, Gilbert DT, Lindzey G, editors. Handbook of Social Psychology. Hoboken, NJ: John Wiley; 2010. p. 353-93.

66. van Stralen D, Mercer TA. During Pandemic COVID-19, the High-Reliability Organization (HRO) Identifies Maladaptive Stress Behaviors: The Stress-Fear-Threat Cascade. Neonatology Today. 2020;15(11):113-24. doi: 10.51362/neonatology.today/2020111511113124.

67. Hediger H. Wild Animals in Captivity. London, UK: Butterworths Scientific Publications; 1950.

68. LeDoux JE. Coming to terms with fear. Proc Natl Acad Sci U S A. 2014;111(8):2871-8. Epub 2014/02/07. doi: 10.1073/ pnas. 1400335111. PubMed PMID: 24501122; PubMed Central PMCID: PMCPMC3939902.

69. LeDoux JE, Pine DS. Using Neuroscience to Help Understand Fear and Anxiety: A Two-System Framework. Am $J$ Psychiatry. 2016;173(11):1083-93. Epub 2016/11/02. doi: 10.1176/appi.ajp.2016.16030353. PubMed PMID: 27609244.

70. van Stralen D, McKay S, Mercer TA. Pragmatic Leadership Practices in Dangerous Contexts: High-Reliability Organizations (HRO) for Pandemic COVID-19. Neonatology Today. 2020;15(8):109-17. doi: 10.51362/neonatology.today/20208158109117.

71. Yates FE. Preface. In: Yates FE, Garfinkel A, Walter DO, Yates GB, editors. Self-organizing systems: The emergence of order. New York, NY Plenum Press 1987. p. xi-xii.

72. Trist EL, Bamforth KW. Some social and psychological consequences of the longwall method of coal-getting: An examination of the psychological situation and defences of a work group in relation to the social structure and technological content of the work system. Human relations. 1951;4(1):338.

73. Clinchy M, Sheriff MJ, Zanette LY. Predator-induced stress and the ecology of fear. Functional Ecology. 2013;27(1):5665.

74. Pyle WH. The Outlines of Educational Psychology: An In- troduction to the Science of Education. Baltimore, MD: Warwick \& York, Inc.; 1912.

75. Haney C, Banks WC, Zimbardo PG. A study of prisoners and guards in a simulated prison. Naval research reviews. 1973;9.

76. Benner P. From Novice to Expert: Excellence and Power in Clinical Nursing Practice. Menlo Park, CA: Addison-Wesley; 1984.

77. Rasmussen J, Vicente KJ. Coping with human errors through system design: implications for ecological interface design. International Journal of Man-Machine Studies. 1989;31(5):517-34. doi: 10.1016/0020-7373(89)90014-x.

78. Reason J. Human Error. New York, NY: Cambridge University Press; 1990.

79. Rasmussen J. What Can Be Learned from Human Error Reports? In: Duncan KD, Gruneberg MM, Wallis D, editors. Changes in Working Life. New York, NY: Wiley; 1980. p. 97-113.

80. Dreyfus SE, Dreyfus HL. The Scope, Limits, and Training Implications of Three Models of Aircraft Pilot Emergency Response Behavior. Berkeley, CA: University of California, Berkeley, Center OR; 1979 February 1979. Report No.: ORC 79-2 Contract No.: ORC 79-2.

81. Dreyfus HL, Dreyfus SE. The Psychic Boom: Flying Beyond the Thought Barrier. Berkeley, CA: University of California, Berkeley, Center OR; 1979 March 1979. Report No.: ORC 79-2.

82. Fisher RA. Cancer and smoking. 1958;182(4635):596-.

83. Fisher RA. Lung cancer and cigarettes? Nature. 1958;182(4628):108.

84. Lifton RJ. Thought Reform and the Psychology of Totalism: A study of brainwashing in China. New York, NY: W. W. Norton \& Co.; 1961.

Disclosures: The authors have no relevant disclosures

NT

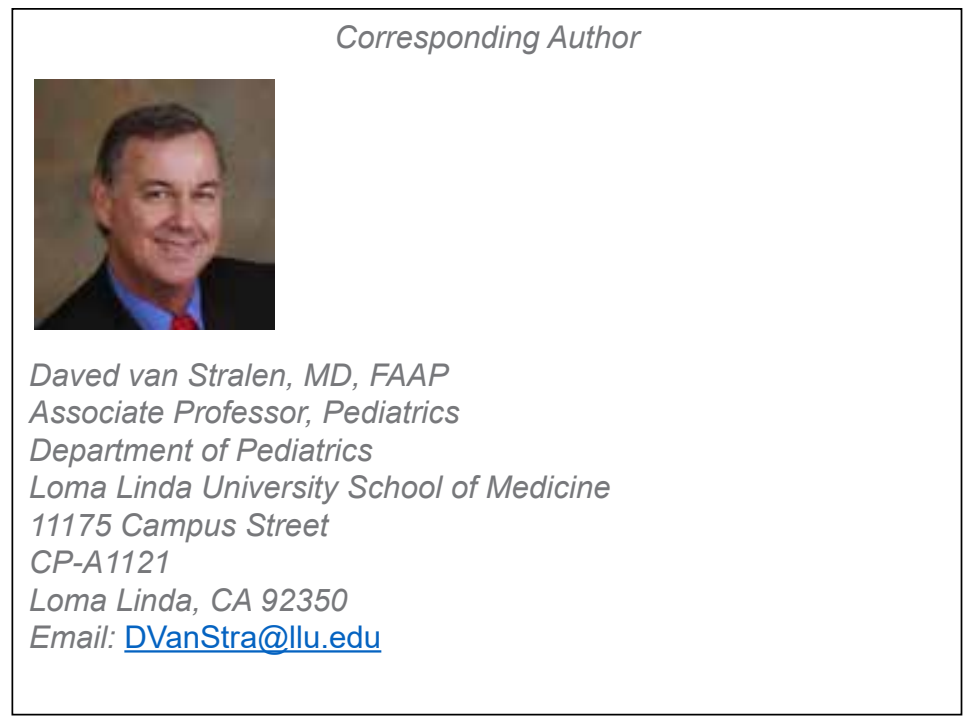

